\title{
Computationally efficient wavefront reconstructor for simulations of multiconjugate adaptive optics on giant telescopes
}

Brent L. Ellerbroek, Luc Gilles, Curtis R. Vogel

Brent L. Ellerbroek, Luc Gilles, Curtis R. Vogel, "Computationally efficient wavefront reconstructor for simulations of multiconjugate adaptive optics on giant telescopes," Proc. SPIE 4839, Adaptive Optical System Technologies II, (7 February 2003); doi: 10.1117/12.459673

Event: Astronomical Telescopes and Instrumentation, 2002, Waikoloa, Hawai'i, United States 


\title{
A Computationally Efficient Wavefront Reconstructor for Simulations of Multi-Conjugate Adaptive Optics on Giant Telescopes
}

\author{
Brent L. Ellerbroek ${ }^{a}$, L. Gilles $^{b}$, and C. R. Vogel ${ }^{c}$ \\ ${ }^{a}$ Gemini Observatory, 670 N. A’ohoku Pl., Hilo, HI 96720 \\ ${ }^{b}$ Michigan Technical University, Dept. of Electrical and Computer Engineering \\ Houghton, MI 49931-1295 \\ ${ }^{c}$ Montana State University, Dept. of Mathematical Sciences \\ Bozeman, MT 59717-2400
}

\begin{abstract}
Multi-conjugate adaptive optical (MCAO) systems with from $10^{4}$ to $10^{5}$ degrees of freedom have been proposed for future giant telescopes. Using standard matrix methods to compute, optimize, and implement wavefront reconstruction algorithms for these systems is impractical, since the number of calculations required to compute (apply) the reconstruction matrix scales as the cube (square) of the number of AO degrees of freedom. Significant improvements in computational efficiency are possible by exploiting the sparse and/or periodic structure of the deformable mirror influence matrices and the atmospheric turbulence covariance matrices appearing in these calculations. In this paper, we review recent progress in developing an iterative sparse matrix implementation of minimum variance wavefront reconstruction for MCAO. The basic method is preconditioned conjugate gradients, using a multigrid preconditioner incorporating a layer-oriented, iterative smoothing operator. We outline the key elements of this approach, including special considerations for laser guide star (LGS) MCAO systems with tilt-removed LGS wavefront measurements and auxiliary full aperture tip/tilt measurements from natural guide stars. Performance predictions for sample natural guide star (NGS) and LGS MCAO systems on 8 and 16 meter class telescopes are also presented.
\end{abstract}

Keywords: Adaptive optics, wavefront reconstruction, extremely large telescopes

\section{INTRODUCTION}

Recent years have seen significant progress in the understanding and design of multi-conjugate adaptive optics (MCAO) systems for wide-field-of-view atmospheric turbulence compensation on large, or 8-10 meter class, telescopes. ${ }^{1}$ An important contributor to this progress has been the availability of analytical methods and detailed simulation tools to optimize wavefront reconstruction algorithms for these systems. Minimum variance wavefront reconstruction methods ${ }^{2}$ permit the first-order effects of the most fundamental AO error sources (fitting error, reconstruction error due to noise, generalized anisoplanatism, and servo lag) to be rapidly and accurately evaluated as a function of basic adaptive optics (AO) system design parameters. These include the deformable mirror (DM) and wavefront sensor (WFS) actuator and subaperture geometries, WFS measurement noise, the configuration of natural- and laser guide stars and the DM conjugate altitudes, and the control loop bandwidth. Minimum variance wavefront reconstruction was originally devised for conventional AO configurations, but a standard formulation for MCAO is now well developed. ${ }^{3-5}$ Wave optics propagation simulations ${ }^{6}$ serve the complementary function of providing detailed analyses of higher-order effects and implementation error sources, such as: Elongated laser guide stars, propagation (diffraction) effects in the atmosphere, optics, and wavefront sensors, DM-to-WFS mis-registration, and non-common path wavefront errors. All of these phenomena may be investigated in detail using existing simulation codes.

Further author information: (Send correspondence to B.L.E)

B.L.E.: E-mail: bellerbroek@gemini.edu, Telephone: 1 (808) 974-2575

C.R.V: E-mail: vogel@gauss.math.montana.edu, Telephone: 1 (406) 994-5332

Adaptive Optical System Technologies II, Peter L. Wizinowich, Domenico Bonaccini, Editors, Proceedings of SPIE Vol. 4839 (2003) @ 2003 SPIE · 0277-786X/03/\$15.00 
In contrast with large telescopes, the current capabilities for detailed analysis and simulation of MCAO on giant (30 meter or larger class) telescopes are fairly limited. The chief obstacle to applying the standard evaluation tools described above is calculating the minimum variance reconstructor in the first place. The computational complexity of this step scales as $O\left(N^{3}\right)$ when employing standard matrix techniques, where $N$ is the order of the AO system. Assuming fixed densities for the DM actuators and WFS subapertures, the computational requirements for calculating the reconstructor for a 32-meter telescope increase by a factor of about $\left[(32 / 8)^{2}\right]^{3}=4096$ relative to the 8 -meter case. The resulting computation time on a high-performance PC or workstation would be completely impractical for MCAO parameters of current interest for giant telescopes.

If this bottleneck in computing the minimum variance reconstructor can be eliminated, the processing requirements for the remaining steps in detailed simulations of MCAO on a (for example) 32 meter telescope are comparatively benign. Propagating wavefronts through atmospheric phase screens is by far the most demanding simulation computation, and the minimum phase screen size that is acceptable for both the 8- and 32-meter cases is set at a common value of about 50-60 meters by the atmospheric outer scale dimension of 20-30 meters. ${ }^{7}$ Fast methods for computing (and if possible applying) minimum variance reconstructors will enable high-fidelity wave optics propagation simulations for 30-meter class MCAO systems with roughly the same computational requirements as the 8-meter case. Developing this modeling capability is an important milestone toward understanding, evaluating, and designing such systems.

Recent progress in developing computationally efficient wavefront reconstruction algorithms for AO systems in general includes work on fast Fourier transform (FFT) algorithms, sparse matrix methods, and iterative preconditioned conjugate gradient (PCG) techniques. ${ }^{8-10}$ All three approaches yield impressive results for extreme AO (ExAO) systems in which a single very-high-order DM is controlled using a single WFS. The computational complexity of computing and applying the reconstructor can be reduced from $O\left(N^{3}\right)$ to $O\left(N^{3 / 2}\right)$ or even $O(N \log N)$. Unfortunately, all three of these methods are either unsatisfactory or inapplicable for the case of $\mathrm{MCAO}$ on giant telescopes due to the more complex structure of the interactions between atmospheric turbulence and WFS measurements. The present version of the FFT reconstructor and the original formulation of the PCG algorithm cannot be used at all. Sparse matrix methods are still usable in principal with complexity $O\left(N^{3 / 2}\right)$, but they become cumbersome for MCAO systems with order $N>3000$ because the sparsity of the turbulence-to-WFS interaction matrix is significantly poorer when multiple sensors and phase screens must be considered. ${ }^{9}$

This paper describes a new version of the iterative PCG wavefront reconstruction algorithm that can be used for MCAO on giant telescopes. Recall that "conjugate gradients" is a standard iterative method for solving linear systems of equations of the form $A x=y$ (as is required for wavefront reconstruction), and that a "preconditioner" is an algorithm for finding an approximate solution to this system that is applied once per iteration to improve the rate of convergence. The new preconditioner is inspired by the layer-oriented MCAO systems and algorithms of current interest, in which each deformable mirror is independently controlled using a wavefront sensor optically conjugated to the identical atmospheric layer. ${ }^{12,13}$ In our new approach, these wavefront measurements are in effect obtained synthetically as linear combinations of multiple WFS measurements recorded in the pupil plane. The three-dimensional atmospheric turbulence profile is related to these synthetic measurements via a block-structured linear system of equations, with the matrix blocks in one-to-one correspondence with the turbulence layers. We use symmetric block Gauss-Seidel (SBGS) iterations as a preconditioner to find an approximate solution to this system. Each phase screen is estimated from the corresponding synthetic WFS measurement using sparse matrix methods that are computationally efficient for the the case of a single atmospheric layer. The SBGS iterations approximately compensate for the cross-coupling between the multiple turbulence layers, much like multiple iterations of the control loop for layer-oriented MCAO.

The remaining features of the approach have been preserved from previous work to improve computational efficiency and increase generality. We use multigrid preconditioning to solve for the turbulence profile on all spatial scales simultaneously and improve the rate of convergence for the low spatial frequency terms. The conjugate gradient algorithm prevents possible stagnation due to poor conditioning of the system of equations that must be solved. All large matrices and matrix-vector operations can be implemented using block-structured sparse matrix representations and techniques, allowing 30-meter class MCAO problems with on the order of $10^{4}$

990 Proc. of SPIE Vol. 4839 
to $10^{5}$ degrees of freedom to be solved on a high-performance PC in a reasonable period of time. The blockstructured representation of the preconditioning computations means that they may be coarsely parallelized with reasonable efficiency in a straightforward fashion. Finally, the complications arising from LGS position uncertainty and the use of natural guide stars to measure full aperture tip/tilt in LGS MCAO systems can be addressed via low-rank matrix perturbations and the use of the Sherman-Morrison matrix inversion lemma.

The remainder of the paper is organized as follows: Section 2 briefly reviews the elements of the minimum variance wavefront reconstruction problem and summarizes one of the standard forms of the solution. Section 3 describes how to improve computational efficiency in the general case via Cholesky factorization of sparse matrices, and section 4 then specializes the general form of the minimum variance reconstructor into the blockstructure representation used here for MCAO. Section 5 outlines how the resulting linear system of equations may be solved using SBGS iterations, and section 6 reviews the basic elements of multigrid methods and the preconditioned conjugate gradient algorithm as used here (Please see our earlier and concurrent papers ${ }^{9-11}$ for further details).

Section 7 summarizes sample numerical results obtained to date for NGS and LGS MCAO systems on 8 to 16 meter class telescopes. Briefly, we find that convergence rates are similar for both NGS and LGS systems and do not degrade appreciably as order of the system increases. Convergence rates do depend on the WFS signal to noise ratio, with more iterations needed to obtain the performance improvements theoretically possible as the measurement noise is reduced. Only a few iterations of the complete conjugate gradient algorithm are needed for convergence at a potentially realistic WFS noise level. The time required (or estimated) per iteration for a $D=16 \mathrm{~m}$ (or $D=32 \mathrm{~m}$ ) MCAO system is about 400 (or 1800) seconds on a $1 \mathrm{GHz}$ Pentium III processor. This is sufficiently efficient to enable simulations of up to a few hundred cycles of the AO control loop, and further improvements should be possible via parallel processing.

\section{MINIMUM VARIANCE WAVEFRONT RECONSTRUCTION}

A brief review of minimum variance wavefront reconstruction is helpful at this point to introduce the necessary notation and to define the linear systems of equations needing to be solved. The purpose of the reconstruction algorithm is to determine a DM actuator command vector $a$ that best compensates for an atmospheric turbulence profile $x$, and minimizes the mean-square phase variance $\sigma^{2}$ associated with the residual wavefront error $\phi$. These quantities are related by the formulas

$$
\begin{aligned}
\phi & =H_{x} x-H_{a} a, \\
\sigma^{2} & =\phi^{T} W \phi .
\end{aligned}
$$

Here $H_{x}$ and $H_{a}$ are the influence matrices that relate the turbulence profile and DM actuator commands to the resulting wavefront in the aperture plane, and $W$ is a positive semidefinite matrix determined by the shape of the aperture. The reconstruction algorithm is a linear operator $E$ applied to the WFS measurement vector s, i.e.

$$
a=E s .
$$

In the open-loop case, the WFS measurement vector $s$ is related to the turbulence profile $x$ by the equation

$$
s=G x+n,
$$

where the matrix $G$ is the turbulence-to-WFS influence matrix, and the vector $n$ is WFS measurement noise. The above model may be formulated to apply to either conventional AO or MCAO systems.

In this notation, the minimum variance reconstructor $E_{*}$ that minimizes the expected value of $\sigma^{2}$ is defined by the condition

$$
E_{*}=\arg \min _{E}\left\langle\sigma^{2}+k\|a\|^{2}\right\rangle .
$$

The angle brackets denote an ensemble average over the statistics of the turbulence profile $x$ and the WFS noise vector $n$, and $k$ is a very small regularizing term included to yield a unique value of $E_{*}$. Using standard least 
squares techniques, ${ }^{5,9}$ the value of the minimum variance reconstructor may be evaluated as

$$
\begin{aligned}
& E_{*}=F_{x} E_{x}, \\
& F_{x}=\left[H_{a}^{T} W H_{a}+k I\right]^{-1} H_{a}^{T} W H_{x}, \\
& E_{x}=\left[G^{T}\left\langle n n^{T}\right\rangle^{-1} G+\left\langle x x^{T}\right\rangle^{-1}\right]^{-1} G^{T}\left\langle n n^{T}\right\rangle^{-1} .
\end{aligned}
$$

The matrix $E_{x}$ provides a minimum-variance estimate of the atmospheric turbulence profile $x$, and the matrix $F_{x}$ determines the best-fit actuator commands for this estimate. The forms of the expressions for the two matrices are analogous, and the primary computational complexity to be avoided is explicit matrix inversion of the two terms appearing within square brackets. For the turbulence estimation operator $E_{x}$ this can be accomplished by first computing the intermediate quantity $y$ defined by

$$
y=G^{T}\left\langle n n^{T}\right\rangle^{-1} s,
$$

and then solving the (symmetric, positive-semi-definite) system of equations

$$
A x=\left(G^{T}\left\langle n n^{T}\right\rangle^{-1} G+\left\langle x x^{T}\right\rangle^{-1}\right) x=y .
$$

for $x$. We will focus attention on the turbulence estimation operator $E_{x}$ for the remainder of this discussion, but all of the methods described below can also be applied to the fitting operator $F_{x}$ with only minor modifications.

\section{SPARSE MATRIX METHODS}

The influence matrices $H_{x}, H_{a}$, and $G$ are quite sparse for many DM and WFS technologies, such as piezo-stack mirrors and Shack-Hartmann wavefront sensors. This fact enables fast implementations of the operators $F_{x}$ and $E_{x}$ using sparse matrix techniques if certain assumptions are made regarding the structure of the matrix $W$ and the statistics of $x$ and $n$. For example, if the noise covariance matrix $\left\langle n n^{T}\right\rangle$ is diagonal and the statistics of $x$ are such that the term $\left\langle x x^{T}\right\rangle^{-1}$ can be neglected, it follows that $G^{T}\left\langle n n^{T}\right\rangle^{-1} G+\left\langle x x^{T}\right\rangle^{-1}=G^{T}\left\langle n n^{T}\right\rangle^{-1} G$ is sparse and can be factored in the form $L L^{T}$, where $\mathrm{L}$ is also sparse and lower triangular.* The computation of $\tilde{x}=E_{x} s$ (where $\tilde{x}$ is the estimate of the true turbulence profile $x$ ) may then be determined efficiently in three steps as follows:

$$
x_{1}=G^{T}\left\langle n n^{T}\right\rangle^{-1} s \quad ; \quad L x_{2}=x_{1} \quad ; \quad L^{T} \tilde{x}=x_{2} .
$$

The last two steps are back substitutions through triangular, sparse matrices. The computational complexity for determining $L$ is $O\left(N^{3 / 2}\right)$, as compared with $O\left(N^{3}\right)$ for the explicit computation of $E_{x}$, yielding significant improvements in speed for AO systems of order as small as $100 .^{14}$

The use of sparse matrix methods (more specifically, minimum degree ordering and Cholesky factorization of sparse matrices ${ }^{15}$ ) for this least squares form of the reconstructor has been known for nearly two decades, but the improvements needed to apply these technique to the general (MCAO) case were developed only last year. ${ }^{9}$ This advance involved finding a sparse approximation to the regularizing term $\left\langle x x^{T}\right\rangle^{-1}$ that does not noticeably degrade reconstruction accuracy, and using the Sherman-Morrison theorem (also known as the matrix inversion lemma) to evaluate the effect of non-sparse, but low rank, modifications to $G$ associated with the tilt uncertainty problem for laser guide stars. With these modifications, sparse matrix methods provide a means of computing and simulating minimum variance reconstructors for very-high-order (or "extreme") conventional AO systems of any dimensionality now being considered. Computation requirements are reduced significantly for MCAO systems as well, although by a smaller factor because the matrix $L$ is appreciably less sparse due to the increased cross-coupling between multiple turbulence layers and guidestars. Reconstructor computations for 16 meter class systems with $N=3000$ or 4000 are relatively straightforward, but the case of $D=32 \mathrm{~m}$ with $N=10000$ remains very cumbersome for the current generation of workstations and PC's. This restriction has as much to do with the limitations of 32-bit memory addressing schemes as with computation times.

\footnotetext{
${ }^{*}$ This is essentially noise-weighted least squares wavefront reconstruction.
} 


\section{BLOCK-STRUCTURED REPRESENTATIONS FOR MCAO}

We have developed a block-structured (or layer oriented) MCAO wavefront reconstruction algorithm to circumvent the limitations of sparse matrix methods as summarized above. To formulate this method, Eq. (4) must be replaced by a more complete model that describes how the WFS measurement vector $s$ depends upon each of the atmospheric turbulence layers. For LGS MCAO, we must also distinguish between LGS and NGS WFS measurements to illustrate how the effects of LGS position uncertainty are are accounted for using low-rank matrix adjustments. The refined formula for $s$ takes the form

$$
\begin{aligned}
s=\left(\begin{array}{c}
s_{h} \\
s_{t}
\end{array}\right) & =\left(\begin{array}{c}
G_{h} \\
G_{t}
\end{array}\right) x+\left(\begin{array}{c}
n_{h} \\
n_{t}
\end{array}\right) \\
& =\left(\begin{array}{ccc}
G_{h, 1} & \cdots & G_{h, L} \\
G_{t, 1} & \cdots & G_{t, L}
\end{array}\right)\left(\begin{array}{c}
x_{1} \\
\vdots \\
x_{L}
\end{array}\right)+\left(\begin{array}{c}
n_{h} \\
n_{t}
\end{array}\right) .
\end{aligned}
$$

Here $s_{h}$ is the higher-order wavefront measurement from the LGS wavefront sensors, $s_{t}$ is the vector of tip/tilt measurements from the full aperture NGS sensors, $n_{h}$ and $n_{t}$ are the noise components of the higher-order and tip/tilt WFS measurements, $x_{l}$ is layer number $l$ of the overall three-dimensional turbulence profile, and $L$ is the number of turbulence layers. $G_{h, l}$ and $G_{t, l}$ are the influence matrices from turbulence layer $l$ to the LGS and NGS WFS measurements, respectively. $G_{h, l}$ is a sparse matrix, and $G_{t, l}$ is a low rank matrix because the dimensionality of $s_{t}$ is small.

The covariance matrices appearing in Eq. (8) for the minimum variance reconstructor can also be described in greater detail for the case of LGS MCAO. Atmospheric turbulence statistics are assumed to be uncorrelated between separate layers, so the inverse of the turbulence profile covariance matrix appearing in Eq. (8) may be written as

$$
\left\langle x x^{T}\right\rangle^{-1}=\operatorname{diag}\left(\left\langle x_{1} x_{1}^{T}\right\rangle^{-1}, \ldots,\left\langle x_{L} x_{L}^{T}\right\rangle^{-1}\right) .
$$

Each diagonal block appearing on the right hand side of Eq (13) may be accurately represented via a sparse approximation as described previously. ${ }^{9}$ Also, the WFS noise covariance matrix takes the form

$$
\left\langle n n^{T}\right\rangle=\left(\begin{array}{ll}
N_{h}+\sigma_{T}^{2} T T^{T} & 0 \\
0 & N_{t}
\end{array}\right)
$$

where $N_{h}$ and $N_{t}$ are diagonal matrices describing the noise in the WFS measurements themselves, $\sigma_{T}^{2}$ is the mean-square position uncertainly for each LGS, and $T$ is a low-rank matrix of 1's and 0's that relates LGS position uncertainty to correlated LGS WFS measurement errors on all of the subapertures of a WFS. In the limit of an infinitely large value for $\sigma_{T}^{2}$, the Sherman-Morrison matrix inversion formula allows the inverse of the WFS noise covariance matrix to be written as

$$
\left\langle n n^{T}\right\rangle^{-1}=\left(\begin{array}{ll}
N_{h}^{-1}-V V^{T} & 0 \\
0 & N_{t}^{-1}
\end{array}\right),
$$

where $V$ is a low-rank matrix with a number of columns equal to twice the number of laser guide stars..$^{9}$ Note that we can consider the case of NGS MCAO as well by setting $V=0$ and defining $s_{t}, G_{t}$, and $n_{t}$ to be null vectors and matrices.

With the above notation, Eq. (9) for $y$ now takes the block-structured form

$$
y_{l}=G_{h, l}^{T}\left(N_{h}^{-1} s_{h}\right)+G_{t, l}^{T}\left(N_{t}^{-1} s_{t}\right)-\left(G_{h, l}^{T} V\right)\left(V^{T} s_{h}\right) \quad l=1, \ldots, L .
$$

Each component $y_{l}$ may be computed efficiently using a combination of diagonal matrix-vector multiplies, sparse matrix-vector multiplies, and low-rank matrix-matrix and matrix-vector multiplies involving matrices ( $V$ and $V^{T}$ ) of low dimension. Next, the linear system $A x=y$ in Eq. (10) may be written as

$$
\left(\begin{array}{ccc}
A_{11} & \cdots & A_{1 L} \\
\vdots & \ddots & \vdots \\
A_{L 1} & \cdots & A_{L L}
\end{array}\right)\left(\begin{array}{c}
x_{1} \\
\vdots \\
x_{L}
\end{array}\right)=\left(\begin{array}{c}
y_{1} \\
\vdots \\
y_{L}
\end{array}\right)
$$


where the blocks of the matrix $A$ are defined by the formulas

$$
A_{k l}= \begin{cases}G_{h, k}^{T} N_{h}^{-1} G_{h, l}+\left[G_{t, k}^{T} N_{T}^{-1} G_{t, l}-\left(G_{h, k}^{T} V\right)\left(G_{h, l}^{T} V\right)^{T}\right] & \text { if } k \neq 1, \\ G_{h, l}^{T} N_{h}^{-1} G_{h, l}+\left\langle x_{l} x_{l}^{T}\right\rangle^{-1}+\left[G_{t, l}^{T} N_{T}^{-1} G_{t, l}-\left(G_{h, l}^{T} V\right)\left(G_{h, l}^{T} V\right)^{T}\right] & \text { if } k=1 .\end{cases}
$$

Each block $A_{k l}$ is a sum of a sparse term or terms, plus a low rank adjustment by the terms grouped above within square brackets. The following section outlines one approach to efficiently finding an approximate solution to this system of equations.

\section{SOLUTION VIA SYMMETRIC BLOCK GAUSS SEIDEL ITERATIONS}

As with several other iterative methods for solving a linear system of equations, SBGS is based upon a "splitting" of the matrix $A$ into diagonal and off-diagonal parts. We write

$$
A=D+L+U,
$$

where the matrices $D, L$, and $U$ represent the diagonal, lower triangular, and upper triangular blocks of the matrix $A$, respectively. With this decomposition, the relationship $A x=y$ is equivalent to either of the formulas

$$
\begin{aligned}
& (D+L) x=y-U x \\
& (D+U) x=y-L x .
\end{aligned}
$$

These relationships may be used to find an approximation solution for $x$, starting with the initial conditions $x(0)=0$ and $y(0)=y=y-U x(0)$ and iterating as follows:

$$
\begin{aligned}
(D+L) x^{\prime}(n+1) & =y(n), \\
y^{\prime}(n+1) & =y-L x^{\prime}(n+1), \\
(D+U) x(n+1) & =y^{\prime}(n+1), \\
y(n+1) & =y-U x(n+1) .
\end{aligned}
$$

The matrix $D+L$ is zero above the (block) diagonal, so Eq. (22) is equivalent to the system of equations

$$
A_{l l} x_{l}^{\prime}(n+1)=y_{l}(n)-\sum_{k<l} A_{l k} x_{k}^{\prime}(n+1) \quad l=1, \ldots, L .
$$

Eq. (26) reduces to $A_{11} x_{1}^{\prime}(n+1)=y_{1}(n)$ for the case $l=1$, which may be solved efficiently for $x_{1}^{\prime}$ using the Sherman-Morrison matrix inversion formula and Cholesky factorization of the sparse component of $A_{11}$ as previously described. ${ }^{9}$ The right-hand-side of $\mathrm{Eq}(26)$ for $l=2$ can then be computed from $y_{2}(n)$ and $x_{1}^{\prime}(n+1)$, which allows $x_{2}^{\prime}(n+1)$ to be determined using the same techniques. After the subvectors $x_{3}^{\prime}(n+1), \ldots, x_{L}^{\prime}(n+1)$ have been computed similarly, Eq. (23) may be evaluated as

$$
y_{l}^{\prime}(n+1)=y_{l}-\sum_{k<l} A_{l k} x_{k}^{\prime}(n+1) \quad l=1, \ldots, L .
$$

The block-structured representations of Eq.'s (24) and (25) are entirely analogous, and the complete SBGS iteration may be implemented in terms of operations involving only sparse and low-rank matrices.

We note that the diagonal blocks $A_{l l}$ appearing on the left-hand-side of Eq. (26) are quite sparse regardless of the number of atmospheric layers $L$, the number of WFS guide stars, or the field-of-view of the MCAO system, since each point on an atmospheric phase screen will only interact with its immediate neighbors regardless of the values of these parameters. Eq. (26) therefore avoids the fill-in and loss of computational efficiency that occurs when the entire system $A x=y$ is solved via a single Cholesky factorization.

Finally, our initial impression is that SBGS should be fairly straightforward to parallelize, given the blockstructured form of the equations listed above.

994 Proc. of SPIE Vol. 4839 


\section{MULTIGRID AND CONJUGATE GRADIENT METHODS}

The SBGS iteration scheme described above will eventually converge to the same phase estimate that would be obtained by explicitly evaluating and applying the minimum variance estimator using conventional matrix techniques. It happens that convergence is most rapid for the high spatial frequency components of the solution, however, and stagnation (very slow convergence) may occur for the low spatial modes of the phase profile that are the dominant terms for a Kolmogorov or von Karman turbulence spectrum. Multigrid methods provide accelerated convergence at low spatial frequencies by (as the name suggests) solving for $x$ at $M$ different spatial scales in series. This process requires representations $A^{(M)}, \ldots, A^{(1)}$ of the operator $A$ at each spatial scale, restriction (or binning) operators $B^{(m)}$ to downsample functions from scale $m$ to scale $m-1$, interpolation operators $I^{(m)}$ for upsampling, and a "smoothing" operator (in this case SBGS iterations) $f\left(A, y, x_{s}\right)$ to find an approximate solution for $A x=y$ beginning with an initial guess $x_{s}$. The steps involved in a single multigrid iteration are briefly as follows:

1. Estimate $x$ on the finest spatial scale as $x^{(M)}=f\left(A^{(M)}, y, 0\right)$.

2. Compute the residual error $r^{(M)}=y-A^{(M)} x^{(M)}$ in the fit to $y$ associated with this estimate of $x$.

3. Downsample this residual to obtain a new value of $y=y^{(M-1)}=B^{(M)} r^{(M)}$ on the next finest grid level.

4. Estimate the downsampled value of $x^{(M-1)}=f\left(A^{(M-1)}, y^{(M-1)}, 0\right)$ on this grid.

5. Repeat steps 2 through 4 until reaching the lowest grid level, and then compute the coarsest solution $x^{(1)}=\left(A^{(1)}\right)^{-1} y^{(1)}$ exactly.

6. Upsample the coarsest solution and sum it with the solution at the next level, updating $x^{(2)}$ as $x^{(2)}+$ $I^{(1)} x^{(1)}$.

7. Apply the smoother $f$ a second time to reduce the high-spatial frequency error induced by the upsampling process, updating $x^{(2)}$ as $f\left(A^{(2)}, y^{(2)}, x^{(2)}\right)$.

8. Iterate steps 6 and 7 until the finest spatial scale is reached.

Note that the interpolation and binning operators $I$ and $B$ may be represented as block-structured sparse matrices, and that the only full matrix-vector multiply required (in step 5) occurs on the coarsest grid where the dimensionality of the vectors involved is small. The computational complexity of a multigrid iteration is consequently defined by the structure of the matrices $A^{(m)}$ and the implementation of the smoothing operator $f$, which in this case consists of sparse matrix-vector multiplies, low rank matrix-vector multiplies, and backsubstitutions through Cholesky factorizations of sparse matrices.

The multigrid algorithm can be applied iteratively in its own right, but convergence may still stagnate depending upon the eigenvalue structure of $A$. Better results may be obtained by using multigrid as a preconditioner for the conjugate gradient algorithm, provided that the operators $B$ and $I$ and the smoother $f$ satisfy certain conditions to insure that the composite operator defined by steps 1-8 above is symmetric. Further details may be found in the companion paper appearing in this volume. ${ }^{11}$

Finally, the rate of convergence will also depend upon the number of SBGS iterations applied in each application of the smoothing operator $f$, the relative size of successive grid levels, and the total number of grid levels $M$. We have determined empirically that good results (in terms of the total computer time required for convergence) for this application are obtained by using a single SBGS iteration, a 2:1 reduction in grid sizes, and a large value for $M$. 
Table 1. Atmospheric turbulence profile used for simulations

\begin{tabular}{|r|r|r|}
\hline Layer & Altitude, $\mathrm{km}$ & Layer weight \\
\hline 1 & 0.00 & 0.652 \\
2 & 2.58 & 0.172 \\
3 & 5.16 & 0.055 \\
4 & 7.73 & 0.025 \\
5 & 12.89 & 0.074 \\
6 & 15.46 & 0.022 \\
\hline
\end{tabular}

\section{SAMPLE NUMERICAL RESULTS}

This section presents performance estimates for a range of sample NGS and LGS MCAO systems on 8 and 16 meter class telescopes. The cases considered form a subset of the scenarios previously evaluated using the sparse matrix implementation of the minimum variance reconstructor. ${ }^{9}$ In revisiting these scenarios we will focus on the convergence behavior of the PCG wavefront reconstruction algorithm, not the performance of the MCAO configurations themselves.

Table 1 summarizes the atmospheric turbulence profile used for these simulations. This profile is a six-layer fit to thermosonde and generalized SCIDAR measurements collected at Cerro Pachon, Chile, the site of the Gemini-South telescope. ${ }^{7}$ The profile has been scaled to obtain a $r_{0}$ of $16 \mathrm{~cm}$ at a wavelength of $0.5 \mu \mathrm{m}$, which corresponds to roughly median seeing. The value of the isoplanatic angle $\theta_{0}$ is $2.65 \mathrm{arc} \mathrm{sec}$, or $12.85 \mu \mathrm{rad}$.

The parameters for the 8- and 16-meter MCAO systems evaluated against this turbulence profile are for the most part borrowed from the baseline design for a MCAO system at Gemini-South. ${ }^{1}$ The field-of-view to be corrected is a one arc minute square. Three deformable mirrors are located conjugate to ranges of $0.0,5.15$, and $10.30 \mathrm{~km}^{\dagger}$, with inter-actuator spacings of $0.5 \mathrm{~m}, 0.5 \mathrm{~m}$, and $1.0 \mathrm{~m}$, respectively. The total number of DM actuators on the three mirrors is 789 for $D=8 \mathrm{~m}$ and 2417 for $D=16 \mathrm{~m}$.

The three DM's are controlled using five higher-order wavefront sensors viewing guidestars at the center and four corners of the 1 arc minute square field of view. The subaperture width is $0.5 \mathrm{~m}$ for each WFS, yielding 224 subapertures per WFS at $D=8 \mathrm{~m}$ and 856 subapertures at $D=16 \mathrm{~m}$. For LGS MCAO the (sodium) guide stars are located at a range of $90 \mathrm{~km}$. In this case, four natural guide stars located at the midpoints of the edges of the field are included for full aperture tip/tilt sensing. ${ }^{\ddagger}$ Two different noise levels of 0.02 and 0.08 arc sec have been considered for the high-order wavefront sensors. The effect of measurement noise is almost negligible for the lower value, while the higher noise value approximately doubles the mean-square phase error averaged over the field. The noise levels for the NGS tip/tilt sensors in the LGS MCAO system are scaled down by the ratio of the subaperture sizes (i.e., a factor of $8 / 0.5=16$ for $D=8 \mathrm{~m}$ and a factor of 32 for $D=16 \mathrm{~m}$ ) to yield the same phase difference measurement error.

Before proceeding to the results themselves, we should note that very similar simulation cases are considered in the companion paper appearing in this volume. ${ }^{11}$ However, there are a variety of modest differences between our separate software implementations that should be recognized when comparing the two papers:

- The simulation code developed for this paper employs either preconditioned conjugate gradients or complete Cholesky factorization for both the turbulence estimation and actuator fitting steps of the reconstructor. The choice can be made separately for each of the two steps, and all of the results presented in this section use PCG for turbulence estimation and complete Cholesky factorization for fitting.

\footnotetext{
${ }^{\dagger}$ This is a modest variation from the Gemini-South values of $0.0,4.5$, and $9.0 \mathrm{~km}$.

${ }^{\ddagger}$ This deviation from the 3 NGS proposed for Gemini-South yields symmetric performance over the square field of view, which simplifies performance analysis.
} 
- The multigrid preconditioner used here consists of 4 levels for the simulations with $D=8 \mathrm{~m}$, and 5 levels for $D=16 \mathrm{~m}$. The smoothing operator consists of a single SBGS iteration (just as in the companion paper).

- The turbulence estimation step computes each atmospheric layer over a region slightly larger than the metapupil, instead of a square domain with a large guard band surrounding the metapupil.

- Boundary effects at the edge of the circular telescope aperture have been modeled more precisely, including the effect on measurements from partially illuminated WFS subapertures.

- Performance evaluation is based on high resolution phase screens with a grid point spacing equal to onesixteenth the DM inter-actuator spacing. This yields a better estimate for the true value of the fitting error, and also models the "spatial aliasing" of high frequency wavefront errors into the WFS measurements.

- The SBGS smoother and multigrid algorithm have been implemented using MATLAB cell arrays to represent block-structured matrices, but the influence matrices $G, H_{x}$, and $H_{a}$ are initially computed as generic sparse matrices. We have not yet upgraded this vestige of our previous sparse matrix code. High resolution simulations of MCAO systems on $D=32 \mathrm{~m}$ telescopes are consequently not yet feasible, since MATLAB presently restricts the product of the dimensions of a sparse matrix to $2^{32}$, regardless of the number of nonzero elements.

Sample results for the $D=8 \mathrm{~m}$ cases are summarized in Fig. 1. The four subplots illustrate the mean-square phase estimation and fitting errors vs. the number of PCG iterations for NGS and LGS MCAO systems and the two different WFS noise levels listed above. The mean-square phase errors have been normalized by the open-loop phase variance, and the results are averages over 10 simulation trials. The performance of the exact minimum variance reconstructor is also plotted for comparison. The PCG algorithm converges to very nearly the exact solution within only one or two iterations at the higher WFS noise level of 0.08 arc sec, while 4-5 iterations are required for convergence at the lower noise level. It appears that convergence is approximately one iteration slower for LGS MCAO than for NGS MCAO, but the distinction is not dramatic.

Fig. 2 illustrates the convergence behavior of the PCG wavefront reconstruction algorithm for simulations of MCAO on 16-meter telescopes. The convergence rates are essentially the same at $D=16 \mathrm{~m}$ as at $D=8 \mathrm{~m}$, and this trend should continue for even larger aperture thanks to the use of a multigrid preconditioner.

Finally, Fig. 3 plots the processing time needed per iteration of the PCG wavefront reconstruction algorithm as a function of the dimensionality of the turbulence profile vector $x$. These times are for a $1 \mathrm{GHz}$ Pentium III processor, and the results obtained for $D=8 \mathrm{~m}$ and $D=16 \mathrm{~m}$ have been extrapolated to the case of $D=32 \mathrm{~m}$ using a power law. LGS MCAO cases require slightly more time than NGS MCAO due to the extra low-rank matrix operations, but this overhead is small and evidently becomes negligible as the dimensionality of the problem increases. Note that $\operatorname{dim}(x) \approx 70,000$ for $D=32 \mathrm{~m}$, and that the time required is approximately one-half hour per PCG iteration. This would enable simulations of 50 to 100 wavefront reconstruction cycles if we are willing to wait 1-2 weeks for results. Faster results should be possible using newer PC's, especially if the SBGS smoother can be parallelized.

\section{ACKNOWLEDGMENTS}

This work was supported by the Gemini Observatory, which is operated by the Association of Universities for Research in Astronomy, Inc., under a cooperative agreement with the NSF on behalf of the Gemini Partnership: the National Science Foundation (United States), the Particle Physics and Astronomy Research Council (United Kingdom), the National Research Council (Canada), CONICYT (Chile), the Australian Research Council (Australia), CNPq (Brazil), and CONICET (Argentina). 
NGS, 0.02 arc sec WFS measurement noise

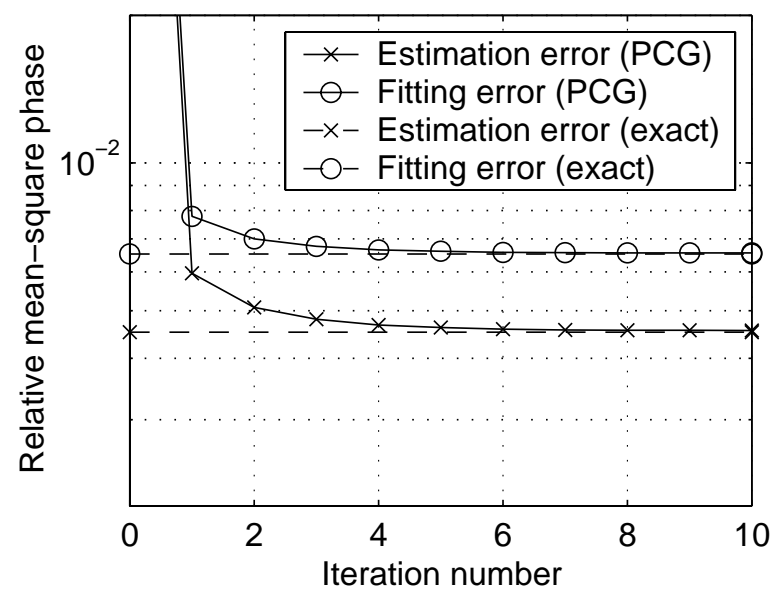

LGS, 0.02 arc sec WFS measurement noise

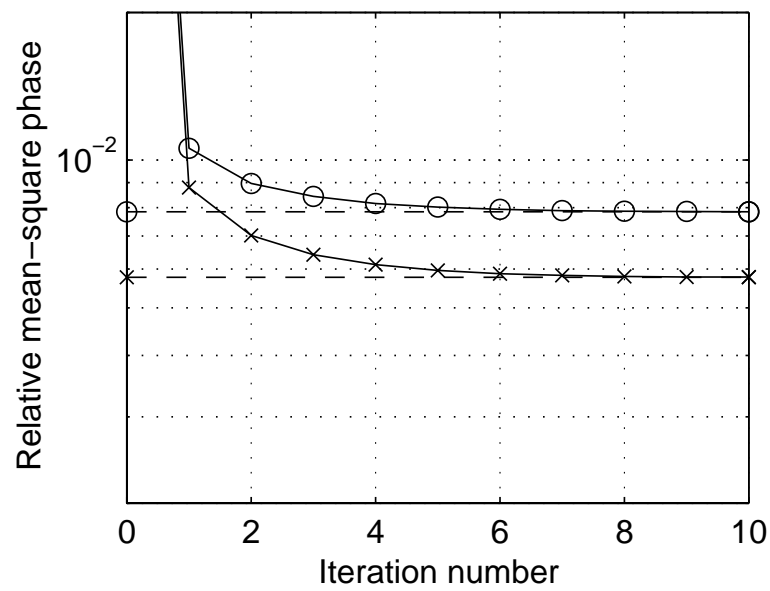

NGS, 0.08 arc sec WFS measurement noise

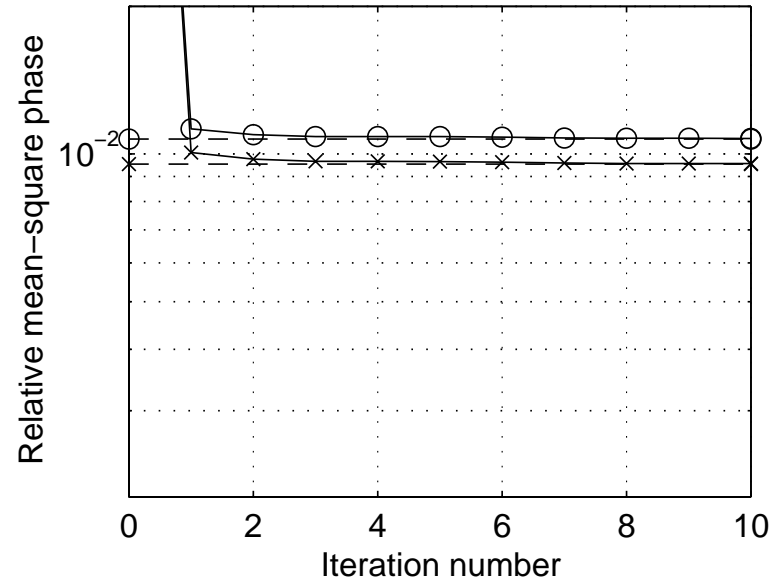

LGS, 0.08 arc sec WFS measurement noise

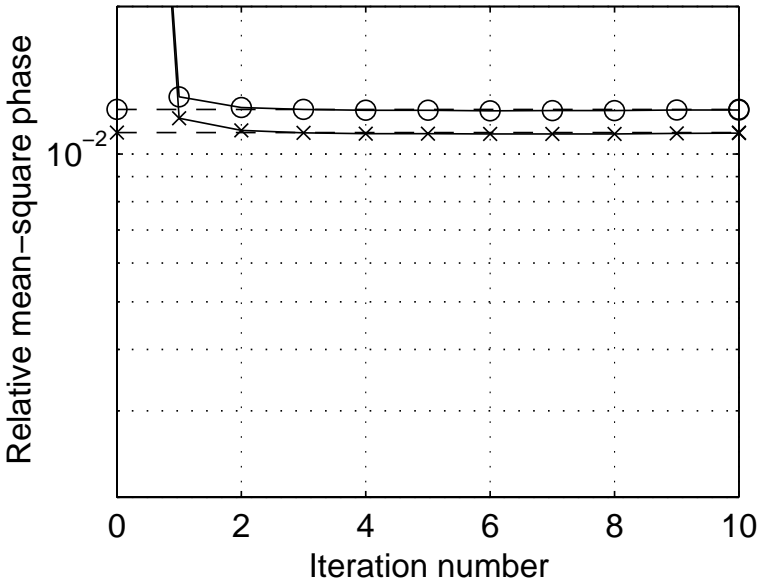

Figure 1. Convergence rates for MCAO with $D=8 \mathrm{~m}$

This figure plots the mean-square phase estimation error vs. the number of preconditioned conjugate gradient (PCG) iterations for order 16 by 16 NGS and LGS MCAO systems and two different levels of WFS measurement noise. The telescope aperture diameter is 8 meters, and the mean-square errors are expressed as a fraction of the open-loop phase variance before AO compensation. For comparison, the dashed baselines plot the mean-square phase estimation and fitting errors for the exact minimum variance turbulence estimator. The numerical range for the vertical axis is $2 \times 10^{-3}$ to $2 \times 10^{-2}$. Further details on the AO system parameters and wavefront reconstruction algorithm may be found in the text.

\section{REFERENCES}

1. Gemini-South MCAO PDR documentation, http://www.gemini.edu/sciops/instruments/adaptiveOptics/AOarchive.html

2. E. P. Wallner, "Optimal wave-front correction using slope measurements," J. Opt. Soc. Am. 73, 1771-1776 (1983).

3. D. C. Johnston and B. M. Welsh, "Analysis of multi-conjugate adaptive optics," J. Opt. Soc. Am. A 11, 394-408 (1994).

4. B. L. Ellerbroek, "First order performance evaluation of adaptive-optics systems for atmospheric turbulence compensation in extended field-of-view astronomical telescopes," J. Opt. Soc. Am. A 11, 783-805 (1994). 

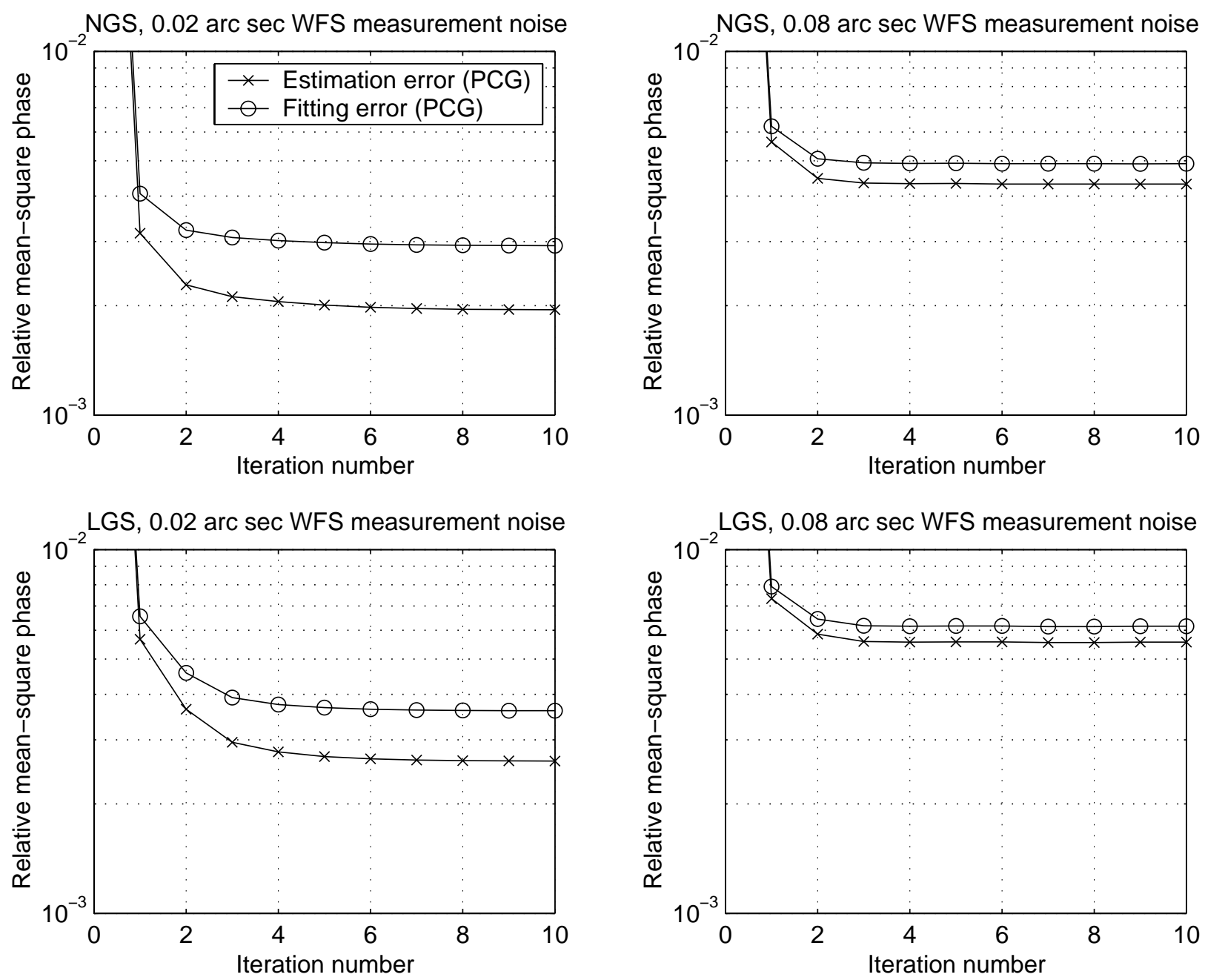

Figure 2. Convergence rates for MCAO with $D=16 \mathbf{m}$

This plot is similar to Fig. 1 above, except that all results correspond to the case of order 32 by 32 MCAO systems on a 16 meter telescope. Only PCG results are presented due to the time required to compute the exact (sparse matrix) wavefront reconstructor.

5. T. Fusco, J.-M. Conan, G. Rousset, L. M. Mugnier, and V. Michau, "Optimal wave-front reconstruction strategies for multi-conjugate adaptive optics," J. Opt. Soc. Am. A 18, 2527-2583 (2001).

6. B. L. Ellerbroek, "Wave optics propagation code for multiconjugate adaptive optics," in Adaptive Optics Systems and Technology II, Robert K. Tyson, Domenico Bonaccini, and Michael C. Roggemann, ed., Proc. SPIE 4494, 104-121 (2001).

7. J. Vernin, A. Agabi, R. Avila, M. Azouit, R. Conan, F. Martin, E. Masciadri, L. Sanchez, and A. Ziad, "1998 Gemini site testing campaign: Cerro Pachon and Cerro Tololo," Gemini Doc. RTP-AO-G0094 (Gemini Observatory, Hilo, Hawaii, 2000).

8. L. A. Poyneer, D. T. Gavel, and J. M. Brase, "Fast wavefront reconstruction in large adaptive optics systems using the Fourier Transform," J. Opt. Soc. Am. A, accepted for publication (2002).

9. B. L. Ellerbroek, "Efficient computation of minimum-variance wave-front reconstructors with sparse matrix techniques," J. Opt. Soc. Am. A, accepted for publication (2002). 


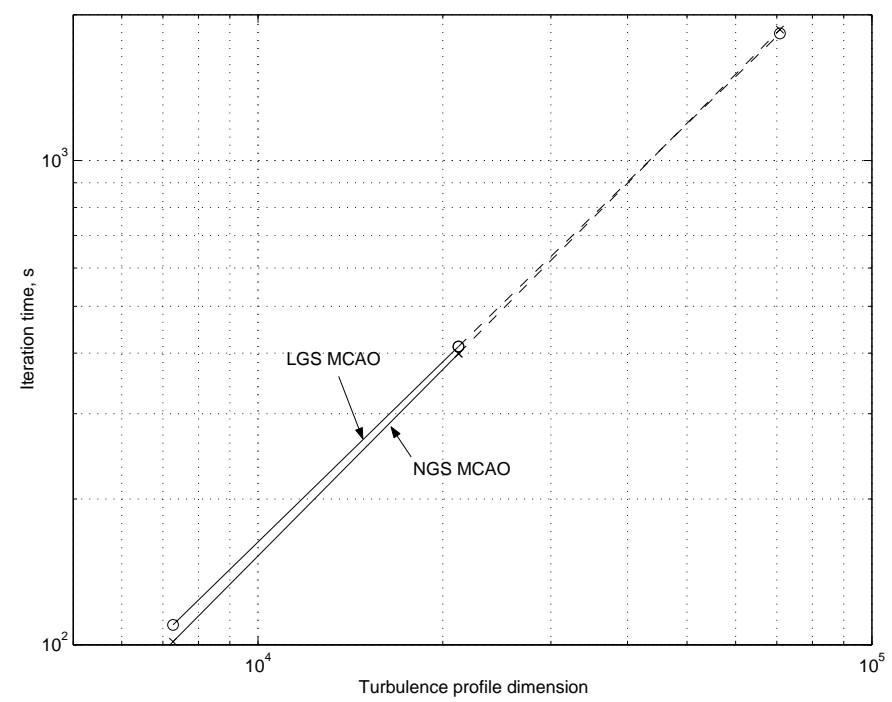

Figure 3. Computation times for the PCG reconstruction algorithm

This figure plots the measured (solid) and extrapolated (dashed) times for one iteration of the PCG wavefront reconstruction algorithm vs. the dimensionality of the atmospheric turbulence profile vector $x$. The times are for a $1 \mathrm{GHz}$ Pentium III processor, and the three data points on each curve correspond to MCAO simulations with aperture diameters of 8 , 16 , and 32 meters.

10. L. Gilles, C. R. Vogel, and B. L. Ellerbroek, "A multigrid preconditioned conjugate gradient method for large scale wavefront reconstruction," J. Opt. Soc. Am. A, accepted for publication (2002).

11. L. Gilles, B. L. Ellerbroek, and C. R. Vogel, "Layer-Oriented Multigrid Wavefront Reconstruction Algorithms for Multi-Conjugate Adaptive Optics," in Adaptive Optical System Technologies II, Peter Wizinowich and Domenico Bonaccini, ed., Proc. SPIE 4839, (2002).

12. R. Ragazzoni, J. Farinato, and E. Marchetti, "Adaptive optics for $100 \mathrm{~m}$ class telescopes: new challenges require new solutions," in Beyond Conventional Adaptive Optics, http://lenin.pd.astro.it/venice2001/proceedings/, Proc. ESO, 2001.

13. M. Tordi, R. Ragazzoni, and E. Diolaiti, "Simulation of a Layer Oriented MCAO system," in Beyond Conventional Adaptive Optics, http://lenin.pd.astro.it/venice2001/proceedings/, Proc. ESO, 2001.

14. G. M. Cochran, "Sparse matrix techniques in wavefront reconstruction," Rep. TR-668 (Optical Sciences Company, Anaheim, Calif, 1986).

15. S. Pissantesky, Sparse Matrix Technology (Academic Press, Orlando, Fla., 1984), Chap. 4. 